\title{
Behandlung der Verbrennungswunde in der Praxis Welche Kriterien dienen der Einschätzung der Wunde?
}

\author{
Treatment of Burns in Medical Practice - \\ What are the Criteria for Assessing a Wound?
}

Autor

Institut

\section{H.-O. Rennekampff}

Sektion für Verbrennungsmedizin und Hautregeneration, Klinik für Plastische, Hand- und Wiederherstellungschirurgie, Medizinische Hochschule Hannover (Direktor: Univ.-Prof. Dr. P. M. Vogt)

\section{Bibliografie}

DOI $10.1055 / \mathrm{s}-2008-1077730$

Akt Dermatol 2008; 34:

422-427 @ Georg Thieme

Verlag KG Stuttgart · New York ISSN 0340-2541

Korrespondenzadresse Univ.-Prof. Dr. Hans Oliver Rennekampff

Sektion für Verbrennungsmedizin und Hautregeneration Klinik für Plastische, Hand- und Wiederherstellungschirurgie Medizinische Hochschule Hannover

Carl-Neuberg-Straße 1 30625 Hannover Rennekampff.Oliver@ mh-hannover.de

\section{Zusammenfassung \\ $\nabla$}

Die kutane Verbrennung stellt eine häufige Verletzung dar. Verbrennungen werden bezüglich der Verbrennungstiefe in 1-, 2a-, 2b-, 3- und 4-gradige Verbrennungen eingeteilt. 2a-gradige Verbrennungen, die nur bis in die oberflächlichen Anteile der Dermis reichen und durch erhebliche Schmerzen und positive Rekapilarisierung erkennbar sind, werden konservativ behandelt. Großflächige Verbrennungen (>15\%-2a-gradig bei Erwachsenen) und Verbrennungen an spe-

\section{Einleitung \\ $\nabla$}

Verbrennungen stellen eine häufige Verletzung dar. Die Gesamtzahl beläuft sich auf eine Verbrennung pro 350 Menschen und Jahr. Kleinste Verbrennungen werden als Bagatelltrauma häufig durch den Patienten selbst behandelt und bedürfen dann oftmals keiner weiteren ärztlichen Behandlung. Hingegen sind großflächigere Verbrennungen, veraltete infizierte Verbrennungswunden, Verbrennungen an der Hand und im Gesicht und insbesondere Verbrennungen bei Kindern mit der notwendigen Sorgfalt zu behandeln, um Folgeschäden wie funktionelle Einschränkungen und Entstellungen durch Narben zu vermeiden.

In dieser Übersicht sollen zum einen die diagnostischen Maßnahmen und die ambulante Behandlung der Verbrennung und zum anderen die Kriterien zur Vorstellung und Behandlung eines brandverletzten Patienten im Verbrennungszentrum dargestellt werden.

\footnotetext{
* Erstveröffentlichung in: Notfall \& Hausarztmedizin 2008; 34 (7): 360-367.
}

ziellen Lokalisationen (Gesicht, Hände, Genitale) sind jedoch stationär zu behandeln. Die konservative Behandlung wird mit topischen Salben und Wundauflagen durchgeführt. Ein Austrocknen des Wundgrundes ist zu vermeiden. Die Reepithelisierung sollte nach 14 Tagen abgeschlossen sein. Infizierte Wunden oder eine ausbleibende Heilung machen eine operative Intervention notwendig. Eine Nachbehandlung mit Narbenpflege und Kompression kann auch bei konservativ behandelten zweitgradigen Verbrennungswunden notwendig sein.

\section{Erstversorgung \\ $\nabla$}

An erster Stelle der Versorgung von Verbrennungsopfern steht die Bergung der Person aus dem Gefahrenbereich. Hierbei müssen die gleichen Vorsichtsmaßnahmen wie bei anderen Unfallopfern angewandt werden. An mögliche Begleitverletzungen, wie sie bei einem Sprung oder Sturz aus großer Höhe oder bei einem Rasanztrauma auftreten können, muss gedacht werden. Gleiches gilt auch bei Verätzungen, Rauchvergiftungen und Stromunfällen. Bei letzteren muss die Entfernung der Noxe unter dem notwendigen Eigenschutz erfolgen.

Galt vor einigen Jahren die sofortige Kühlung der Brandwunde noch als Qualitätsmerkmal der Erstversorgung, besteht heute Einigkeit, dass eine Kühlung nur noch bei kleinen Verletzungen ( $<10 \%$ der Körperoberfläche) bei nicht intubierten Patienten sinnvoll ist [6]. Die Kühlung kann dabei das lokale Ödem initial günstig beeinflussen und die Patienten empfinden eine temporäre Schmerzlinderung. Die thermische Schädigung der Haut ist durch die Kühlung jedoch nicht reversibel zu beeinflussen. Zur Kühlung sollte nur Flüssigkeit mit einer Temperatur von $>12^{\circ}$ Celsius verwendet werden [9]. Die Kühlung mit Eis oder Eiswasser ist zu vermeiden, da dies zu einer 
Tab. 1 Verbrennungsgrade.

\begin{tabular}{|llllll|}
\hline $\begin{array}{l}\text { Verbrennungsgrad } \\
\text { Aussehen }\end{array}$ & $\mathbf{1}$ & $\mathbf{2 a}$ & $\mathbf{2 b}$ & $\mathbf{3}$ & $\mathbf{4}$ \\
& Rötung & Blasenbildung & Blasenbildung & Brandschorf & $\begin{array}{l}\text { verbrannte Haut und } \\
\text { Gewebe, freiliegende } \\
\text { tiefere Strukturen }\end{array}$ \\
\hline Wundgrund & keine Wunde & rötlich, feucht & $\begin{array}{l}\text { weißlich, rot, teils } \\
\text { thrombosierte Venen }\end{array}$ & $\begin{array}{l}\text { weiß, bräunlich } \\
\text { lederartig }\end{array}$ & $\begin{array}{l}\text { Denaturierung, } \\
\text { Verkohlung }\end{array}$ \\
\hline Rötung wegdrückbar & ja & ja & kaum/nein & nein & nein \\
\hline Berührungsschmerz & deutlich & sehr deutlich & herabgesetzt & fehlt & fehlt \\
\hline Nadelstichblutung & sofort & sofort & gering & keine & keine \\
\hline & konservative Behandlung & operative Behandlung & & &
\end{tabular}

weiteren zellulären Schädigung führt. Lediglich bei Verätzungen sollte eine intensive Spülung der Wunde mit Wasser erfolgen, da in den meisten Fällen eine spezifische lokale Therapie mit Neutralisation der Noxe nicht möglich ist. Eine Ausnahme stellt die Verätzung mit Flusssäure dar. Sie wird topisch und intraläsional mit Kalziumglukonat behandelt. Eine ausreichende Tetanus-Immunisierung muss geprüft werden. Im Zweifelsfall erfolgt eine aktive und passive Tetanusimpfung.

\section{Anamnese}

Das Erheben der Unfallanamnese ist von weiter reichender Bedeutung. So kann ein Verbrennungstrauma im geschlossenen Wohnraum bereits anamnestisch auf ein zusätzliches Inhalationstrauma hinweisend sein. Auch der Hinweis auf die Noxe gibt einen wichtigen Hinweis auf die zu erwartende Schädigung. Heißes Fett enthält eine größere thermische Energie als kochendes Wasser, und die Immersion in kochendes Wasser überträgt mehr Energie als Wasserdampf oder heiße Luft. Bei Verätzungen ist die Anamnese mit Hinweis auf die Noxe für eine mögliche weitere spezifische Therapie von Bedeutung. Bei Verbrennungen im Kindesalter hat die Anamnese im Vergleich zum Verbrennungsmuster eine weiter reichende Bedeutung, um eine eventuelle Kindesmisshandlung aufzudecken. Nicht zuletzt ist die Anamnese wichtig, um Arbeitsunfälle von Privatunfällen abzugrenzen und eine geeignete Vorstellung einzuleiten.

\section{Befundung \\ $\nabla$}

Charakteristischerweise wird die Verbrennungswunde in vier Grade eingeteilt ( $\bullet$ Tab. 1).

\section{Verbrennungsgrad 1}

Grad 1 entspricht einer Hyperämie, die durch ein Erythem erkennbar ist. Es findet sich ein lokales Ödem und Schmerzen.

\section{Verbrennungsgrad 2}

Grad 2a oder die oberflächlich zweitgradige Verbrennung ( $\triangle$ Abb. 1) stellt eine Verbrennung mit geringer Tiefenausdehnung, aber mit Verlust der Epidermis und oberer Anteile der Dermis dar.

Meist findet sich eine Blasenbildung und der Wundgrund weist eine wegdrückbare Hyperämie auf (Rekapilarisierungs-Test positiv). Der Wundgrund ist sehr schmerzhaft. Hautanhangsgebilde in tieferen Anteilen der Dermis sind intakt. Grad 2b, oder die tief zweitgradige Verbrennung ( $\bullet$ Abb. 2) hat eine größere Tie-

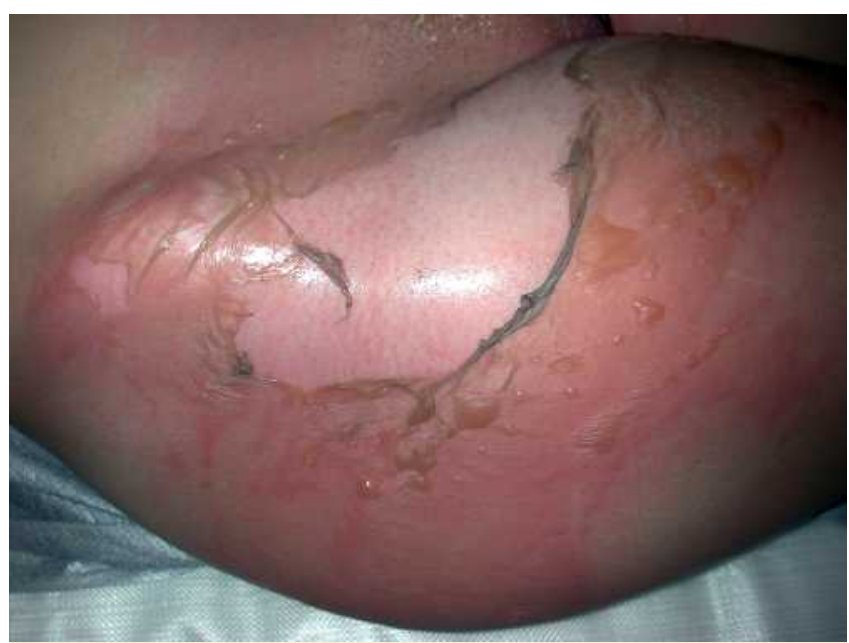

Abb. 1 Verbrennung 2a: Deutlich zu erkennen ist der hyperämische, feuchte Wundgrund.

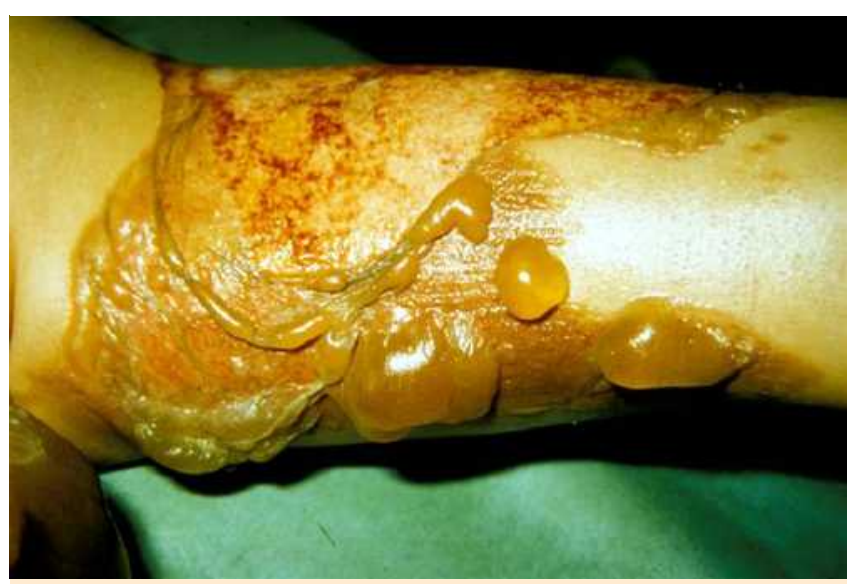

Abb. 2 Verbrennung 2b: Im Gegensatz zu der oberflächlich zweitgradigen Verbrennung finden sich teils dunkelrote, teils thrombosierte Areale.

fenausdehnung in die Dermis, ebenfalls mit Verlust der Epidermis, aber auch mit weitgehendem Verlust der Hautanhangsgebilde. Auch hier kann eine Blasenbildung auftreten.

Der Wundgrund ist teils dunkel gerötet, teils weiß, die Rötung ist nicht wegdrückbar (negativer Rekapilarisierungs-Test). Die Dermis ist nicht durchblutet. Es besteht eine geringe bis fehlende Schmerzhaftigkeit. 


\section{Verbrennungsgrad 3}

Grad 3 oder die drittgradige Verbrennung ( $\odot$ Abb. 3 und - Abb. 4) stellt eine Verbrennung dar, bei der alle Hautschichten zerstört sind.

Der Wundgrund ist weiß bis lederartig, die Schmerzempfindung ist aufgehoben, und eine Durchblutung ist nicht mehr nachweisbar.

\section{Verbrennungsgrad 4}

Bei Grad 4 oder der Verkohlung sind neben der Haut auch Anteile der Unterhaut, Faszien oder Muskel betroffen und thermisch schwer geschädigt.

\section{Einschätzung der Verbrennungstiefe}

Die Einschätzung der Verbrennungstiefe erfolgt klinisch anhand der Parameter Rekapilarisierung und Schmerzhaftigkeit. Während die Einschätzung in eine oberflächliche Grad-2a-Verbrennung ( $\odot$ Abb. 1) und drittgradige Verbrennung ( $\odot$ Abb. 3) in aller Regel nur wenig Erfahrung erfordert, ist die Befundung der

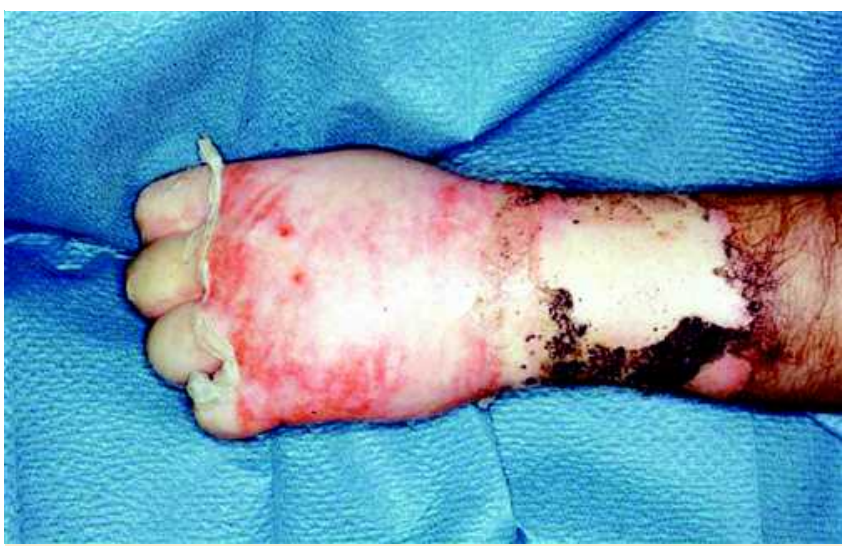

Abb. 3 Drittgradige Verbrennung am distalen Unterarm mit weißem Wundgrund. Der Nadelstichtest und Rekapilarisierungstest sind in diesem Areal negativ. Am Handrücken findet sich eine tief zweitgradige Verbrennung; die Verbrennung reicht bis in tiefe Anteile der Dermis; es findet sich noch partiell ein roter Wundgrund; eine Rekapilarisierung ist aber bereits nicht mehr feststellbar.

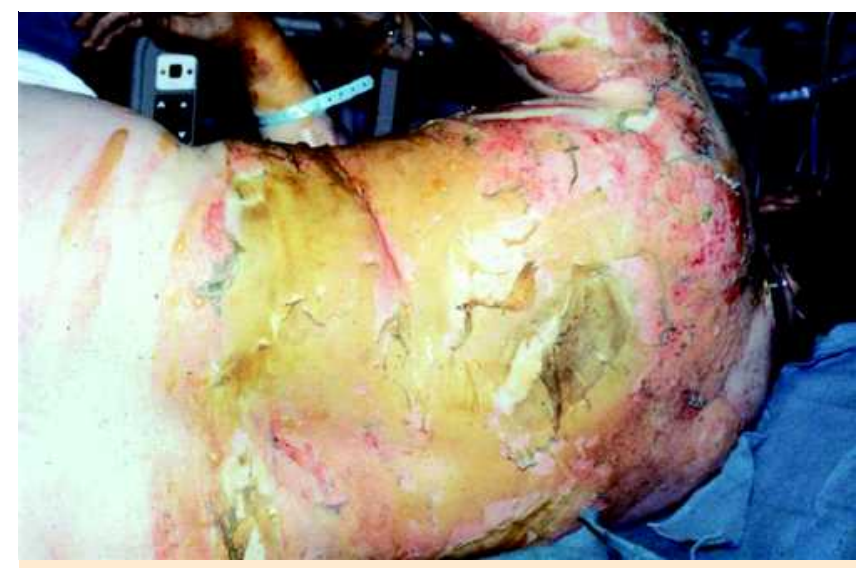

Abb. 4 Der charakteristische lederartige Wundgrund ohne Schmerzempfindung bei einer drittgradigen Verbrennung am Rücken vor der Wundreinigung.
Verbrennungstiefe $2 \mathrm{~b}$ und korrekte Abgrenzung von der oberflächlichen 2a-gradigen Verbrennung an die Erfahrung des Untersuchers gebunden $(\bullet$ Abb. $\mathbf{5})$.

Diese Abgrenzung ist von erheblicher therapeutischer Bedeutung, da 2a-Verbrennungen konservativ behandelt werden, 2b-Verbrennungen aber bereits einer chirurgischen Therapie bedürfen. Präzise objektivierbare diagnostische Methoden wie Laserdopplerflowmetrie [1] oder die histologische Untersuchung stehen für die Befundung im hausärztlichen Bereich nicht zur Verfügung.

\section{Bestimmung der Verbrennungsfläche}

Die Bestimmung der Verbrennungsfläche kann anhand zweier Regeln erfolgen: Zum einen gilt, dass die Handfläche des Patienten $1 \%$ seiner Körperoberfläche entspricht. Eine weitere Möglichkeit ist die Einschätzung der Verbrennungsfläche nach der Neunerregel nach Wallace, wobei beim Erwachsenen den Körperregionen Anteile von $9 \%$ der Gesamtkörperoberfläche zugeordnet werden $(\bullet$ Abb. $\mathbf{6})$.

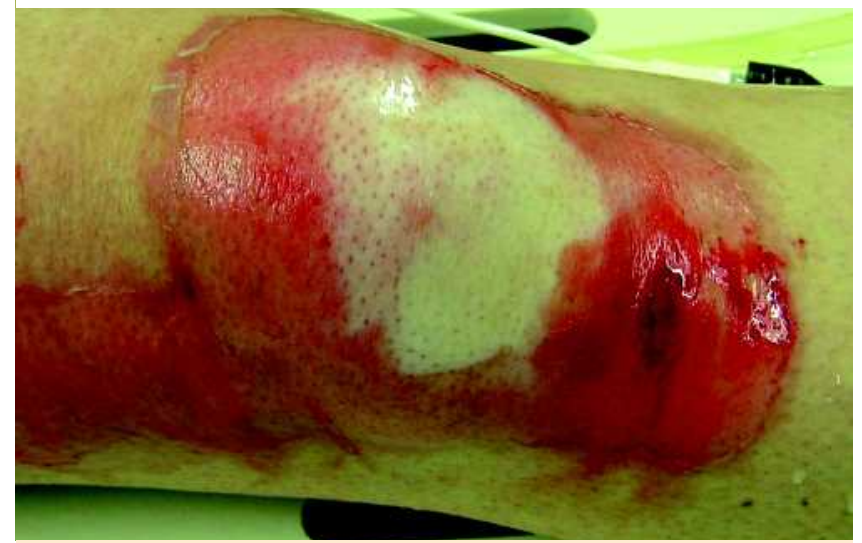

Abb. 5 Bei dieser Verbrennung am Knie zeigt sich nach Entfernung der Blasen in den mittleren Anteilen ein weißlicher Wundgrund mit reduzierter Schmerzhaftigkeit, entsprechend einer tief zweitgradigen Verbrennung. Die umgebenden Areale mit rötlichem Wundgrund und guter Rekapilarisierung sind als oberflächlich zweitgradig einzustufen.

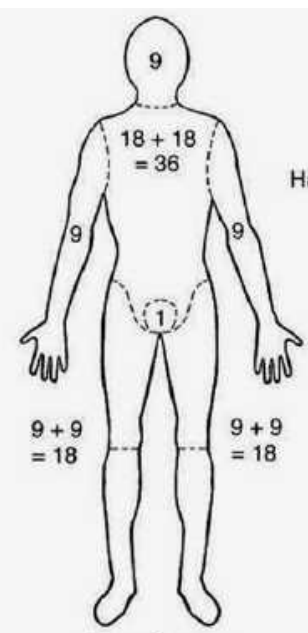

Erwachsener

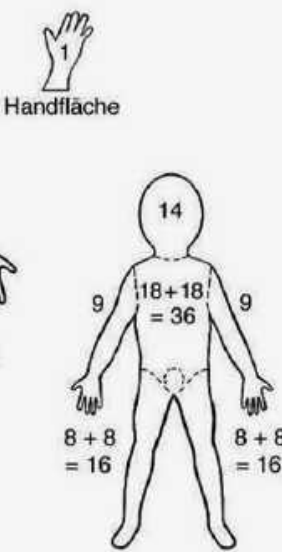

Kind/5 Jahre

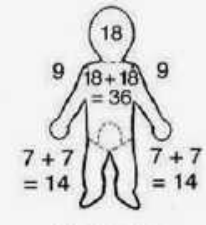

Kind/1 Jahr
Abb. 6 Neunerregel nach Wallace. 
Bei der Berechnung der verbrannten Gesamtkörperoberfläche werden erstgradige Verbrennungen nicht miteingerechnet.

\section{Behandlung \\ $\nabla$}

Generell gilt, dass die erstgradige Verbrennung, das Erythem, und die oberflächlich zweitgradige Verbrennung (2a) einer konservativen Behandlung zugeführt werden. Tief zweit- und drittgradige Verbrennungen erfordern eine operative Intervention mit Nekrektomie und autologer Hauttransplantation, sodass eine stationäre Einweisung in eine chirurgische Klinik notwendig sein wird. Obgleich die meisten chirurgischen Kliniken eine Hauttransplantation durchführen können, kann es sinnvoll sein, den Patienten direkt in ein Verbrennungszentrum einzuweisen. Besonders bei großflächigen Verbrennungen kann der Umweg über das nahe gelegene Krankenhaus Gefahren wie Unterkühlung oder eine inadäquate Flüssigkeitstherapie mit sich bringen, sodass die direkte Einweisung in ein Verbrennungszentrum als sinnvoll erachtet wird. Dazu kann eine direkte Kontaktaufnahme mit dem nächstgelegenen Verbrennungszentrum oder eine Vermittlung über die zentrale Vermittlung für Schwerbrandverletzte in Hamburg (Tel.: 040/42851-3998) erfolgen. In jedem Fall stehen die Verbrennungszentren mit ihrer Notfallaufnahme jederzeit beratend und gegebenenfalls zur Aufnahme zur Verfügung.

\section{Kriterien für die stationäre Einweisung in ein Verbrennungszentrum \\ $\nabla$}

Bei dem in Tab. 2 gelisteten Ausmaß der Verbrennung hat die Fachgesellschaft eine Behandlung (Deutsche Gesellschaft für Verbrennungsmedizin) [10] in einem Verbrennungszentrum empfohlen.

\begin{tabular}{|c|c|}
\hline $\begin{array}{l}\text { Verbrannte Körperoberfläche } \\
\text { (KOF) }\end{array}$ & $\begin{array}{l}>15 \% \text { KOF } 2^{\circ} \text { oder }>10 \% 3^{\circ} \text { bei } \\
\text { Erwachsenen, }<10 \% \text { KOF bei Kindern }\end{array}$ \\
\hline Tiefe der Verbrennung & $\begin{array}{l}2 \mathrm{~b}^{\circ}-3^{\circ} \text { bei entsprechender } \\
\text { Ausdehnung }\end{array}$ \\
\hline Lokalisation & $\begin{array}{l}\text { Gesicht, Hals, Hände, Genitale, } \\
\text { Achselhöhlen }\end{array}$ \\
\hline Allgemeinzustand & $\begin{array}{l}\text { Alter unter } 8 \text { Jahre bzw. über } 60 \text { Jahre, } \\
\text { schwerwiegende Grunderkrankung }\end{array}$ \\
\hline Verletzungsart & $\begin{array}{l}\text { Inhalationstrauma } \\
\text { Elektroverbrennung }\end{array}$ \\
\hline
\end{tabular}

\section{Stromverletzungen \\ $\nabla$}

Ein besonderes Augenmerk gilt den Stromverletzungen [5]. Hier ist die Lichtbogenverletzung von dem tatsächlichen Stromdurchtritt durch den Körper durch eine genaue Anamnese und eventuell durch das Vorhandensein von Ein- und Austrittsmarken zu unterscheiden. Während es sich bei der Lichtbogenverletzung letztlich um eine thermische Schädigung der Haut handelt, ist bei der Verbrennung beziehungsweise Verletzung durch einen Stromdurchtritt immer von einer weit über das Hautniveau reichenden Verletzung von Muskel, Gefäßen und Nerven auszugehen. Oftmals täuscht die Stromeintritts- und -austritts-

marke eine viel kleinere Schädigung vor. Daher müssen Stromverletzungen stationär behandelt werden und sollen in Verbrennungszentren vorgestellt werden.

\section{Transport}

$\nabla$

Für den Transport wird der Patient in eine metallisierte Rettungsdecke oder Brandwundenverbandtücher eingeschlagen, bei kleinen Verbrennungen ist das Anlegen eines sterilen Verbandes ausreichend. Die Anwendung spezieller Verbandsstoffe und Wundauflagen ist für einen kurzen Transport nicht notwendig.

\section{Ambulante Versorgung \\ $\nabla$}

\section{Initiale Schmerzbehandlung}

Die initiale Schmerzbehandlung erfolgt mit intravenös fraktioniert applizierten Opioiden wie zum Beispiel Piritramid. Eine intramuskuläre oder subkutane Medikamentengabe ist ebenso wie die orale Zufuhr in der akuten Notfallsituation zu vermeiden. An eine ausreichende Schmerzmedikation ist aber auch bei der ambulanten Weiterbehandlung zu denken. Gerade die Verbrennungen, die keiner chirurgischen Therapie bedürfen, nämlich die oberflächlichen zweitgradigen Verbrennungen, stellen die schmerzhaftesten Verbrennungen dar. Die Verschreibung einer oralen Schmerzmedikation, zum Beispiel mit Tramadol oder Tilidin, erfolgt individuell.

\section{Débridement der Brandblasen}

Ist die Entscheidung für die ambulante hausärztliche Weiterbehandlung gefallen, erfolgt die lokale Wundbehandlung. Hierzu werden die Brandblasen abgetragen, sofern dies nicht bereits für die Beurteilung der Wunde geschehen ist. Das Abtragen der Blasen und insbesondere der entleerten Brandblasen hat eine prophylaktische Bedeutung [7]. Zum einen wird der Wundgrund vom Druck der hyperonkotischen Brandblasenflüssigkeit entlastet und zum anderen werden die schädigenden Einflüsse der Brandblasenflüssigkeit auf die Wunde entfernt. Geplatzte oder lediglich eröffnete Brandblasen stellen ein hohes Risiko für die Keimbesiedlung dar, sodass Brandblasen komplett débridiert werden sollten.

\section{Antimikrobielle Behandlung}

Nach diesem Débridement der Brandblasen und der aseptischen Reinigung des Wundgrundes wird eine topische Wundbehandlung der zweitgradigen oberflächlichen Verbrennung durchgeführt ( Tab. 3). Deren Ziel ist die antimikrobielle Abschirmung der Wunde und Optimierung des Wundmilieus mit Beschleuni-

Tab. 3 Wundauflagen und Salben für die ambulante Behandlung.

antimikrobielle Salben enzymatische Salben

Alginate mit Silber

Hydrokolloid mit/ohne Silber

Fettgaze mit/ohne antimikrobielle Wirkstoffe Spezialfolien antibakterielle Abschirmung der Wunde oberflächliches Débridement der Wunde mit Verbesserung der Epithelisierung antibakteriell und feuchtigkeitsregulierend feuchtigkeitsregulierend, ggf. mit antibakterieller Abschirmung Wundgrundprotektion, ggf. mit antibakterieller Abschirmung Verbesserung der Epithelisierung 
gung der Reepithelisierung. Für die topische antimikrobielle Behandlung der Wunde steht eine Reihe von Salben zur Verfügung [8].

Flammazine ${ }^{\circledR}$-Salbe (Silbersulfadiazin) wird in Kombination mit einer Fettgaze verwendet. Flammazine ${ }^{\circledR}$ hat eine gute Wirksamkeit gegenüber vielen Mikroorganismen. Nachteilig ist der zytotoxische Effekt auf die Reepithelisierung und das Entstehen eines Pseudoschorfes aus Salbe und Exsudat. Dieser Pseudoschorf kann leicht eine tiefere Verbrennungstiefe oder eine Infektion der Wunde vortäuschen.

Repithel ${ }^{\circledR}$ stellt eine Kombination aus Hydrogel und Polyvinylpyrrolidone-jodid in Liposomen dar. Repithel ${ }^{\circledR}$ besitzt weite antimikrobielle und fungizide Eigenschaften. Zudem wird die Rehydrierung der Wunde günstig beeinflusst. Nachteilig sind die hohen Kosten.

An weiteren Möglichkeiten stehen Chlorhexidin oder Fusidinsäure in Kombination mit Salbe oder Salbenkompressen zur Verfügung. Alternativ können auch kollagenasehaltige Salben Verwendung finden; besonders dann, wenn dünne oberflächliche Beläge von denaturiertem Kollagen abgetragen werden sollen. Tägliche Verbandwechsel mit Kontrolle der Wundverhältnisse schließen sich bis zur Reepithelisierung an. Auch silberhaltige Alginatverbände können in der Behandlung von oberflächlich zweitgradigen Verbrennungen angewendet werden. Okklusive Verbände mit Hydrogelplatten oder neuartigen Milchsäurederivaten (Suprathel ${ }^{\circledR}$ ) erfordern Erfahrung beziehungsweise sind sehr teuer.

Ein evidenzbasierter Vergleich der unterschiedlichen Materialien für die Verbrennungsbehandlung fehlt. In jedem Fall ist aber ein Austrocknen des Wundgrundes zu vermeiden, da dies zu einem Abtiefen der Verbrennungstiefe führt und eine zeitgerechte Heilung behindert. In gleicher Weise gilt das Gerben der Verbrennungswunde heute als obsolet.

\section{Reevaluation der Verbrennungswunde}

Die Reevaluation der Verbrennungswunde sollte am Folgetag des Verbrennungstraumas durchgeführt werden, um ein Abtiefen der Verbrennung zu erkennen. Ein prolongierter Zellschaden durch das thermische Trauma kann dazu führen, dass eine initial als oberflächlich zweitgradig eingestufte Verbrennung in der Reevaluation dann tiefer erscheint und bereits einer tief zweitgradigen Verbrennung entspricht. Bei der weiteren Abheilung ist auch darauf zu achten, dass es nicht zur Infektion der Verbrennungswunde kommt. Die lokale Infektion der Verbrennungswunde kann zum einen zu einer systemischen Infektion führen, zum anderen führt die Infektion der Wunde zu einer Gewebeschädigung mit konsekutiver Vertiefung der Wunde. Dennoch kann eine prophylaktische Antibiose nicht generell empfohlen werden. Sie sollte dem Fall der nachgewiesenen Infektion vorbehalten bleiben. Im Fall einer Infektion der Verbrennungswunde muss ein zusätzliches chirurgisches Débridement erfolgen, da systemische Antibiotika nicht die nekrotischen Gewebeanteile der Verbrennungswunde erreichen.

\section{Abheilungszeiten}

Die Heilung der oberflächlichen zweitgradigen Verbrennungswunde ist in der Regel nach 14 Tagen erfolgt. Nur im Ausnahmefall kann eine Reepithelisierungsdauer bis 21 Tage toleriert werden. Bei Abheilungszeiten die darüber hinausgehen, muss von einer initialen Fehleinschätzung der Verbrennungstiefe oder einem Nachtiefen der Verbrennungswunde ausgegangen werden. Ist die Wunde nach dieser Zeit nicht abgeheilt, muss ein chirurgisches Débridement und eine Hauttransplantation erfolgen. Ein längeres Warten führt zu einer übermäßigen hypertrophen Narbenbildung [3]. Eine Hauttransplantation ist in diesen Fällen mit weniger Narbenbildung verbunden und deshalb anzustreben.

\section{Nachbehandlung}

$\nabla$

Der Nachbehandlung kommt eine besondere Bedeutung zu, da alle Verbrennungswunden, bei der tiefere Anteile der Dermis geschädigt sind und/oder die durch eine Hauttransplantation versorgt wurden, eine mehr oder weniger starke Narbenbildung zeigen. Hierbei gilt in der Regel, dass die Zeit bis zum Wundverschluss und der Verlust dermaler Anteile mit einer stärkeren Narbenbildung korrelieren [2]. Nach Abschluss der konservativen oder operativen Therapie erfolgt die Verordnung und Anpassung von Kompressionswäsche, wie zum Beispiel Kompressionshandschuhen, Kompressionsärmlingen oder Kompressionshosen. Die Kompressionsbehandlung soll der Entstehung hypertropher Narben entgegenwirken. Auch Blutumlaufstörungen und der Juckreiz in Narben werden günstig beeinflusst. Die Kompression wird bis zum zweiten Jahr nach dem Trauma als sinnvoll erachtet.

Der oft starke Juckreiz der Narben kann durch topische kortisonhaltige Cremes wie zum Beispiel Linola $\mathrm{HN}^{\circledR}$ gemildert werden. Bei kleineren Arealen kann die Auflage von Silikonplatten, zum Beispiel Acante Gelfolie ${ }^{\circledR}$ oder CicaCare ${ }^{\circledR}$ einen günstigen Effekt haben. In gleicher Weise steht Silikon auch als topisches Gel zur Verfügung. Der Effekt von so genannten Narbencremes ist wissenschaftlich derzeit nicht belegt. Für spezielle Fragen der rekonstruktiven und ästhetischen Nachbehandlung nach einer Verbrennung stehen die Verbrennungszentren mit ihren Nachsorge-Sprechstunden zur Verfügung.

\section{Abstract \\ Treatment of Burns in Medical Practice - What are the Criteria for Assessing a Wound? $\nabla$}

Burn injuries are a common trauma. Burn injuries to the skin are divided according their depth into erythema, superficial partial thickness, deep partial thickness and full thickness wounds. Superficial partial thickness wounds are characterized by intense pain and a positive blanching test. Superficial partial thickness wounds of up to $10 \%$ total body surface area can be treated as out-patients. Large burns and those at critical areas like hand, face and genitalia should be treated in surgical departments or may require treatment at specialized burn centers. Superficial partial thickness burns are treated topically with antimicrobial ointments or wound dressings. It is of major importance to avoid desiccation of the wound bed. Reepithelialization should be completed by day 14. Infection of the wound or a non-healing wound makes surgical intervention necessary. In the case of postburn scarring silicon dressings and compression garments are necessary. 


\section{Literatur}

1 Chatterjee JS. A critical evaluation of the clinimetrics of laser doppler as a method of burn assessment in clinical practice. J Burn Care Res 2006; 27: $123-130$

2 Cubison TC, Pape SA, Parkhouse N. Evidence for the link between healing time and the development of hypertrophic scars (HTS) in paediatric burns due to scald injury. Burns 2006; 32: 992 - 999

3 Deitch EA, Wheelahan TM, Rose MP et al. Hypertrophic burn scars: analysis of variables. J Trauma 1983; 23: $895-898$

4 Granick M, Boykin J, Gamelli $R$ et al. Toward a common language: surgical wound bed preparation and debridement. Wound Repair Regen 2006; 14 Suppl 1: $1-10$
5 Hülsbergen-Krüger S, Pitzler D, Partecke BD. Hochspannungsunfälle, Besonderheiten und Behandlung. Unfallchirurg 1995; 98: 218-223

6 Lönnecker S, Schoder V. Hypothermia in patients with burn injuries: Influence of prehospital treatment. Chirurg 2001; 72: 164-167

7 Sargent RL. Management of blisters in the partial-thickness burn: an integrative research review. J Burn Care Res 2006; 27: 66-81

8 Vasel-Biergans A, Probst (Hrsg.)Wundauflagen. Stuttgart: Wissenschaftliche Verlagsgesellschaft mbH, 2003:

9 Venter TH, Karpelowsky JS, Rode H. Cooling the burn wound: the ideal temperature of the coolant. Burns 2007; 33: 917-922

10 www.verbrennungsmedizin.de

Buchbesprechung

Patch Testing. Test Concentrations and Vehicles for

4350 Chemicals

De Groot AC

Wapserveen, Niederlande: acdegroot publishing, 3. Aufl., 2008.

456 S., geb. 129,95€

ISBN 978-90-813233-1-4

Professor de Groot, als Dermatologe und Allergologe Hochschullehrer an der Universitätshautklinik Groningen/Niederlande und vornehmlich bekannt als Autor zahlreicher Publikationen unter Betonung allergologischer Themen wie z.B. „Unerwünschte Arzneimittelreaktionen“ oder „Die allergische Kontaktdermatitis“, präsentiert in einer dritten, um 650 neue Kontaktallergene erweiterten Auflage ein in dieser Form einmaliges Werk, in dem 4350 Testsubstanzen mit - seit der letzten Auflage von 1994 überwiegend neu hinzugefügten - 2200 Synonymen aufgelistet sind.

Wie auch die vorherige ist die aktuelle dritte Auflage in $7 \mathrm{Ka}-$ pitel untergliedert. Im umfangreichen ersten Kapitel sind in insgesamt 6 Spalten jeweils Testsubstanzen in alphabetischer Reihenfolge mit den dazugehörigen Synonymen und den entsprechenden, von mehreren Autoren empfohlenen Testkonzentrationen und Vehikeln aufgeführt. Dazugehörige Literaturhinweise sind anschließend in Klammern dargestellt. Weiterhin findet der Leser die entsprechende Merck Index Nummer, die die jeweilige Testsubstanz ausweist. Durch das Aufführen des Zeichens „+“" erkennt der Leser, dass die korrespondierende Testsubstanz aus Spalte 1 in der CTFA Cosmetic Ingredient Dictionary and Handbook (11. Auflage, 2006) dargestellt ist. Abschließend erhält der Leser in der sechsten Spalte wesentliche Informationen zur Testprozedur der einzelnen Substanzen wie z. B. „mögliche irritative Reaktionen“, „Risiko einer Sensibilisierung durch die Testsubstanz im Epikutantest“ oder „zu geringe Anzahl von Kontrollpersonen“.

Im zweiten Kapitel listet der Autor in alphabetischer Reihenfolge 196 gängige Abkürzungen der Testsubstanzen neben ihren Eigennamen auf. Kapitel 3 enthält Informationen über Test- konzentrationen und Vehikel für Testsubstanz-Gruppen, die entweder aufgrund ihrer chemischen Struktur oder Funktionalität in Beziehung zueinander stehen und somit kreuzreaktives Potenzial besitzen. Im vierten Kapitel wird der Leser über Testkonzentrationen und Vehikel für Produkte wie z. B. feste Materialien, Nahrungsmittel, organische Lösungen u.a. informiert. Kapitel 5 enthält eine Auflistung von Testsubstanzen mit fotosensitivem Potenzial und im sechsten Kapitel werden Testsubstanzen aufgeführt, die für die Auslösung von Kontaktreaktionen vom Soforttyp bekannt sind. Zum Abschluss findet der Leser im siebten Kapitel eine Auflistung potenzieller Sensibilisatoren im Epikutantest. „It is easy to patch test but it is not so easy to arrive at correct conclusions“ - ein Aphorismus, der auch aktuell Gültigkeit besitzt.

Nach der ersten Auflage 1986 und der Zweiten 1994 liefert Herr Professor de Groot nun eine inhaltlich noch reichere, aktualisierte Auflage mit Bezug zu zeitgenössischen Literaturquellen. Sein Werk dient nicht als Leitfaden, sondern als gut strukturierte, hervorragende Informationsquelle für den kritischen Anwender der Epikutantestung, der frei darüber entscheiden kann, welcher Empfehlung er folgt.

Um 650 neue Testallergene erweitert, enthält die jetzige Auflage eine Fülle an schnell nachschlagbaren, wertvollen Informationen zur Kontaktdermatitis. Sie ist jedem allergologisch tätigen Dermatologen unbedingt zu empfehlen und eine gute Investition, nicht zuletzt weil die Suche nach entsprechenden Testkonzentrationen und Vehikeln enorm erleichtert wird. Dem Autor gebührt großer Dank für dieses Werk.

Hanan Adib-Tezer, Wiesbaden 